\title{
Role of Hepatic Lipase and Endothelial Lipase in High-Density Lipoprotein-Mediated Reverse Cholesterol Transport
}

\author{
Wijtske Annema • Uwe J. F. Tietge
}

Published online: 22 March 2011

(C) The Author(s) 2011. This article is published with open access at Springerlink.com

\begin{abstract}
Reverse cholesterol transport (RCT) constitutes a key part of the atheroprotective properties of high-density lipoproteins (HDL). Hepatic lipase (HL) and endothelial lipase (EL) are negative regulators of plasma HDL cholesterol levels. Although overexpression of EL decreases overall macrophage-to-feces RCT, knockout of both HL and EL leaves RCT essentially unaffected. With respect to important individual steps of RCT, current data on the role of EL and HL in cholesterol efflux are not conclusive. Both enzymes increase hepatic selective cholesterol uptake; however, this does not translate into altered biliary cholesterol secretion, which is regarded the final step of RCT. Also, the impact of HL and EL on atherosclerosis is not clear cut; rather it depends on respective experimental conditions and chosen models. More mechanistic insights into the diverse biological properties of these enzymes are therefore required to firmly establish EL and HL as targets for the treatment of atherosclerotic cardiovascular disease.
\end{abstract}

Keywords Atherosclerosis - Bile - Cardiovascular disease . Cholesterol · Efflux · Endothelial lipase · Feces · Hepatic lipase $\cdot$ High density lipoproteins $\cdot$ Inflammation · Liver . Macrophage $\cdot$ Macrophage-to-feces · Phospholipase ·

Reverse cholesterol transport · Risk · Therapy · Triglycerides

W. Annema $\cdot$ U. J. F. Tietge $(\bowtie)$

Department of Pediatrics, Center for Liver, Digestive, and Metabolic Diseases, University Medical Center Groningen, University of Groningen,

Hanzeplein 1,

9713 GZ, Groningen, The Netherlands

e-mail: u_tietge@yahoo.com

W. Annema

e-mail: w.annema@med.umcg.nl

\section{Introduction}

Despite the widespread use of low-density lipoprotein (LDL) reduction therapies, atherosclerotic cardiovascular disease (CVD) remains a leading cause of morbidity and mortality in developed countries. Treatment with statins (HMG-CoA reductase inhibitors), for example, only decreases the risk of major coronary events by $15 \%$ to $40 \%$ even though these drugs consistently and efficiently lower LDL cholesterol [1]. This substantial residual risk indicates a potential clinical benefit of novel therapeutic strategies that look beyond LDL and focus on high-density lipoproteins (HDL). Plasma HDL cholesterol levels are strongly, inversely, and independently related to cardiovascular disease risk [2]. A major part of this atheroprotective potential of HDL conceivably consists of its key role in reverse cholesterol transport (RCT, see below for details) [2]. Among the factors modulating HDL metabolism, lipases such as hepatic lipase (HL) and endothelial lipase (EL) are of prime importance. HL and EL both belong to the triacylglycerol lipase family, which also includes lipoprotein lipase (LPL), but members of this family differ in their respective hydrolytic activities. Whereas LPL is almost exclusively a triglyceridase, HL uses both phospholipids and triglycerides as substrates, whereas EL possesses predominantly phospholipase and very little triglyceridase activity (Table 1) [3]. By decreasing the triglyceride and phospholipid content of HDL, HL and EL are both significant negative regulators of plasma HDL levels [2]. Therefore, pharmacologic inhibition of these lipases might represent a valid approach to increase HDL cholesterol. This review focuses on the impact of both HL and EL on HDL metabolism and RCT, but also addresses the consequences of modulating $\mathrm{HL}$ and EL expression and activity for atherosclerotic lesion development. 
Table 1 Characteristics of hepatic lipase and endothelial lipase

\begin{tabular}{|c|c|c|}
\hline & Hepatic lipase & Endothelial lipase \\
\hline Structural features & $65-\mathrm{kD}$ glycoprotein & 68-kD glycoprotein \\
\hline Tissue expression & Hepatocytes, macrophages & $\begin{array}{l}\text { Endothelial cells, macrophages, thyroid gland, } \\
\text { hepatocytes, kidney, lung, ovary, testis, and placenta }\end{array}$ \\
\hline Lipolytic activity & $\mathrm{TG}>\mathrm{PL}$ & $\mathrm{PL}>\mathrm{TG}$ \\
\hline Substrates & All lipoproteins & HDL \\
\hline Impact on plasma HDL cholesterol levels & $\begin{array}{l}\text { Knockout } \uparrow \\
\text { Overexpression } \downarrow\end{array}$ & $\begin{array}{l}\text { Knockout } \uparrow \\
\text { Overexpression } \downarrow\end{array}$ \\
\hline Impact on cholesterol efflux & Knockout $\uparrow \leftrightarrow$ & $\begin{array}{l}\text { Knockout } \downarrow \uparrow \\
\text { Overexpression } \uparrow \downarrow\end{array}$ \\
\hline Impact on hepatic uptake of HDL cholesterol & $\begin{array}{l}\text { Knockout } \downarrow \\
\text { Overexpression } \uparrow\end{array}$ & $\begin{array}{l}\text { Knockout } \downarrow \\
\text { Overexpression } \uparrow\end{array}$ \\
\hline Impact on biliary cholesterol secretion & Knockout $\leftrightarrow$ & Overexpression $\leftrightarrow$ \\
\hline Impact on fecal neutral sterol and bile acid excretion & Knockout $\leftrightarrow$ & Overexpression $\leftrightarrow$ \\
\hline Impact on macrophage-to-feces RCT & Knockout $\leftrightarrow$ & $\begin{array}{l}\text { Knockout } \leftrightarrow \\
\text { Overexpression } \downarrow\end{array}$ \\
\hline Impact on atherosclerosis & $\begin{array}{l}\text { Knockout } \uparrow \downarrow \\
\text { Overexpression } \downarrow \\
\text { Macrophage expression } \uparrow \\
\text { Macrophage knockout } \uparrow\end{array}$ & Knockout $\downarrow \leftrightarrow$ \\
\hline
\end{tabular}

$T G$ triglycerides, $P L$ phospholipids, $R C T$ reverse cholesterol transport; $\uparrow=$ increased; $\downarrow=$ decreased; $\leftrightarrow=$ not affected. Multiple arrows per condition indicate divergent reports in literature

\section{Role of High-Density Lipoproteins in Reverse Cholesterol Transport}

HDL plays a central role in RCT (Fig. 1) [2]. The first step in RCT involves the efflux of cholesterol from peripheral cells, most importantly macrophage foam cells within atherosclerotic plaques, toward either apolipoprotein (apo) A-I, which is mainly ATP-binding cassette transporter A1 (ABCA1)-mediated, or HDL, a process mainly driven by ABCG1. Lecithin:cholesterol acyltransferase (LCAT) then catalyzes the formation of mature spherical HDL by converting free cholesterol to apolar cholesterol ester, which moves to the core of the HDL particle. In addition to $\mathrm{ABCA} 1$ and $\mathrm{ABCG1}$, the scavenger receptor class $\mathrm{B}$ type I (SR-BI) might also contribute to net cholesterol efflux. The cholesterol originating from peripheral cells is subsequently taken up into the liver in several distinct pathways that are all relevant for RCT: 1) selective uptake mediated by SR-BI, 2) holoparticle uptake involving the extracellularly localized $\beta$-chain of the mitochondrial F1 ATPase as well as P2Y13 receptors, and 3) in species such as humans, rabbits, hamsters, and monkeys, but not mice and rats, transfer to apoB-containing lipoproteins by cholesteryl ester transfer protein (CETP) followed by uptake via LDL receptors. The major pathway for disposal of hepatic cholesterol from the body is generally believed to be its secretion into bile in the form of either free cholesterol or bile acids. Eventually, neutral sterols (cholesterol and its metabolites) and bile acids are excreted via the feces. The RCT pathway has been reviewed in more detailed elsewhere [2].

\section{Hepatic Lipase}

$\mathrm{HL}$ is a $65-\mathrm{kD}$ glycoprotein synthesized and secreted primarily by hepatocytes and to a lesser extent by macrophages (Table 1) [4]. In the liver, HL is anchored via heparan sulfate proteoglycans to hepatocytes in the Space of Disse and to the sinusoidal endothelial cell surface [4]. Virtually all lipoprotein subclasses are substrates for HL, resulting in the generation of smaller and denser particles (Table 1) [5]. In addition, independent of its catalytic activity, HL anchored to cell surface proteoglycans also has a bridging function promoting receptor-mediated uptake of lipoproteins [4].

\section{HL and HDL Metabolism}

By hydrolyzing triglycerides and phospholipids, HL converts lipid-rich large $\mathrm{HDL}_{2}$ to smaller, more dense $\mathrm{HDL}_{3}$. Mice lacking HL characteristically display increased plasma levels of larger, phospholipid- and triglyceride-enriched 


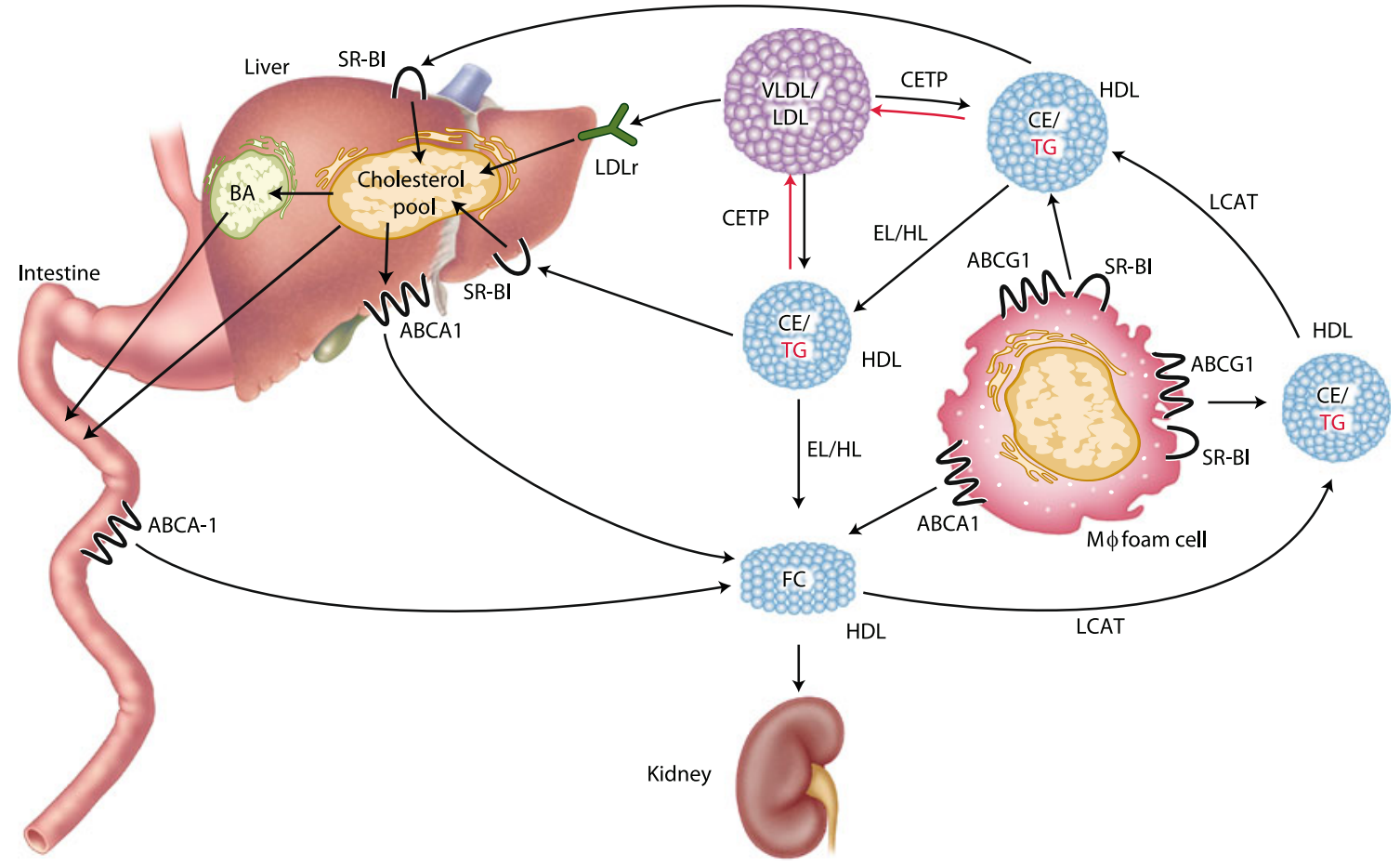

Fig. 1 Schematic depicting the impact of hepatic lipase (HL) and endothelial lipase (EL) on reverse cholesterol transport (RCT). Small discoidal high-density lipoprotein (HDL) particles are generated by the liver (about $70 \%$ contribution) and by the intestine (about $30 \%$ contribution). Free cholesterol (FC) from macrophage foam cells is effluxed toward these particles by ATP-binding cassette transporter A1 (ABCA1). Through the action of lecithin:cholesterol acyltransferase (LCAT), these particles mature and become spherical; ABCG1 and scavenger receptor class B type I (SR-BI) add more cholesterol onto these larger HDL. EL and HL hydrolyze HDL phospholipids and phospholipids/triglycerides (TG), respectively, thereby destabilizing the particle, resulting in shedding of poorly lipidated apolipoprotein (apo) A-I that is subject to clearance by the kidneys. Cholesteryl ester from these HDL particles becomes more susceptible towards SR-BImediated selective uptake. In turn, selective uptake is also required for HDL remodeling by these lipases to continue. Via SR-BI, HDL

HDL (Table 1) [6•]. In turn, in mice and in rabbits, overexpression of human HL resulted in a marked decrease in HDL cholesterol due to an increased catabolic rate [5]. HL can also impact on HDL metabolism independent of its lipolytic properties, as adenovirus-mediated overexpression of catalytically inactive HL in HL knockout mice led to a reduction in plasma HDL levels, though to a lesser extent than observed in mice overexpressing similar levels of wild-type HL [5]. HL deficiency in humans also results in elevated levels of larger $\mathrm{HDL}_{2}$ particles, an enrichment of HDL with triglycerides, and hyperalphalipoproteinemia attributable to slower catabolism of apoA-I [7]. Consistently, certain polymorphisms in the HL gene such as S267F, C-480T, and C-514T that result in lower HL expression and activity are firmly associated with higher plasma HDL cholesterol $[8,9]$. These combined data support a key role for $\mathrm{HL}$ as a negative regulator of plasma HDL levels. cholesterol enters the hepatic cholesterol pool and can either be directly secreted as free cholesterol into bile and feces or after metabolic conversion into bile acids. Cholesteryl ester transfer protein (CETP), expressed in humans but not in mice and rats, mediates the hetero-exchange of cholesteryl ester (CE) originating from HDL with triglycerides originating from apoB-containing lipoproteins. TGenrichment of HDL makes the particle a better substrate for HL. On the other hand, cholesterol transferred to apoB-containing lipoproteins can be taken up into the liver via low-density lipoprotein receptors (LDLR) and then also enters the hepatic cholesterol pool. EL and HL expression consistently results in lower plasma HDL levels, increased hepatic cholesterol content, but unchanged biliary cholesterol secretion rates. Results regarding the impact of these lipases on cholesterol efflux are variable. The net effect of the absence of HL and EL on overall RCT is minimal, whereas overexpression of EL has been shown to decrease RCT. VLDL - very low-density lipoprotein

\section{Impact of HL on Selected Key Steps in RCT}

Cholesterol efflux is a critical first step in RCT [2]. Plasma as well as HDL from HL-deficient mice has an increased capacity to elicit cholesterol efflux from lipid-laden macrophages in vitro (Table 1), which was ascribed to phospholipid enrichment of HDL from the HL knockouts [6•]. However, in humans with a complete deficiency of HL, the ability of plasma or isolated HDL to promote cholesterol efflux from normal human skin fibroblasts was not altered [7]. Thus, the impact of HL on macrophage cholesterol efflux remains to be elucidated. It would be interesting to also study the effect of HL overexpression, because HL increases the formation of pre $\beta$-HDL, an important mediator of ABCA1-mediated macrophage cholesterol efflux [5]. In addition, the impact of macrophage HL on cholesterol efflux has not been explored yet. 
Another important step in RCT is hepatic uptake of HDL cholesterol, and several lines of evidence indicate that HL stimulates this process (Table 1). HL increases the selective uptake of HDL cholesteryl ester (CE) in hepatic and nonhepatic cell lines [4]. Moreover, in vivo hepatic selective uptake of HDL-CE was impaired in HL knockout mice [6•] and significantly higher in mice overexpressing human $\mathrm{HL}$ [5]. Both the hydrolytic activity of HL and its heparan sulfate proteoglycan-dependent bridging properties contribute to the stimulating effect of HL on SR-BI-mediated selective uptake [5].

Although HL facilitates the uptake of HDL cholesterol into the liver, this does not appear to translate into increased biliary sterol secretion (Table 1), a third important step in RCT. The biliary secretion rates of cholesterol and bile acids were identical in HL-deficient mice and wild-type controls, even when fed cholesterol-enriched or lithogenic diets [10]. Consequently, HL deficiency did not change fecal neutral sterol or bile acid excretion (Table 1) [10].

\section{HL and Macrophage-Specific RCT}

Even though RCT from macrophages to feces represents only a small fraction of the total cholesterol pool, this flux is nevertheless considered highly relevant for the development of atherosclerosis. When mice were injected with ${ }^{3} \mathrm{H}$ cholesterol-labeled macrophages into the peritoneal cavity and the appearance of label in plasma and feces was followed for $48 \mathrm{~h}$, plasma levels of ${ }^{3} \mathrm{H}$-sterol were higher in mice deficient in HL compared to controls. However, overall macrophage-to-feces RCT determined by excretion of macrophage-derived label into the feces over $48 \mathrm{~h}$ remained essentially unaffected in HL knockouts (Table 1) [6•]. On the other hand, the effects of HL overexpression have not been addressed thus far. Also, HL expression by macrophages might have an impact on RCT that has not been experimentally explored yet.

\section{HL and Atherosclerosis}

Several studies have thus far addressed the role of HL in atherosclerosis development in different rodent models (Table 1) and humans but without reaching conclusive results. In LDL receptor-knockout mice, aortic atherosclerotic lesion formation was accelerated when endogenous HL was also absent [11], whereas transgenic overexpression of human HL reduced atherosclerosis by $40 \%$ to $70 \%$ $[12,13]$. Overexpressing the catalytically inactive variant of human HL was atheroprotective in chow-fed LDL receptor-knockout mice lacking endogenous HL [13, 14], whereas no effect on atherosclerosis development was seen in the same model on a Western diet [12]. In contrast, HL deficiency in apoE knockout mice on a regular chow diet reduced aortic lesion size by $75 \%$ despite increased circulating levels of atherogenic apoB-containing lipoproteins. Decreased atherosclerosis development in this mouse model was proposed to be due to higher HDL levels and an increased capacity of HDL to elicit cholesterol efflux [15]. Furthermore, HL deficiency delayed the onset of occlusive atherosclerosis, resulting in myocardial infarction and cardiac dysfunction in SR-BI/ apoE double-knockout mice [16]. However, the impact of $\mathrm{HL}$ on atherosclerotic lesion formation in an experimental animal model expressing CETP has not been reported. Bone marrow transplantation studies showed that restricting HL expression to macrophages enhanced the formation of early atherosclerotic lesions in both apoE knockout mice and LCAT transgenic mice without changing plasma lipoprotein levels [17]. On the other hand, a more recent publication demonstrated that deficiency of leukocytederived HL reduced circulating HDL cholesterol levels and increased the extent of aortic atherosclerosis in LDL receptor-knockout mice expressing human CETP [18]. Whether the experimental design or the mouse model used offers the explanation for these divergent results remains to be seen.

Complete HL deficiency in humans is a rare condition; however, some of these patients develop premature coronary artery disease despite increased plasma HDL cholesterol levels [19]. Likewise, a lower post-heparin plasma HL activity has been related to a higher extent of coronary artery disease in 200 men undergoing coronary angiography [20]. In contrast, human studies investigating the relationship between polymorphisms in the HL gene, which supposedly resulted in reduced HL activity, and atherosclerotic cardiovascular disease did not provide a clear picture (summarized in Table 2). In an autopsy cohort of 700 middle-aged Finnish men, the HL C-480T promoter polymorphism was related to a larger coronary atherosclerotic plaque area [21], and in a large population-based Danish study homozygosity for three common single nucleotide polymorphisms in the HL promoter $(-216 \mathrm{~A}$, $-480 \mathrm{~T}$, and $-729 \mathrm{G}$ ) was associated with an increased risk of ischemic heart disease despite higher plasma HDL cholesterol [22]. Furthermore, the prevalence of the $\mathrm{C}$ to $\mathrm{T}$ substitution at position 514 of the HL gene was significantly higher in 490 middle-aged male Caucasian Australian patients with coronary heart disease compared with 330 controls [23]. Controversially, in the almost 9000 participants of the Copenhagen City Heart study, no association between risk for ischemic cardiovascular disease and six genetic variants of the HL gene was detectable [8]. Importantly, the frequency of the $-514 \mathrm{~T}$ allele of HL was identical between men with documented coronary artery disease and healthy controls [24]. Other researchers found in a multiethnic population that individuals with the $\mathrm{CC}$ 
Table 2 Selected hepatic lipase and endothelial lipase gene polymorphisms and their association with atherosclerosis and cardiovascular disease

\begin{tabular}{|c|c|c|c|c|c|c|}
\hline Lipase & $\begin{array}{l}\text { Gene } \\
\text { polymorphism }\end{array}$ & $\begin{array}{l}\text { Number of } \\
\text { subjects }\end{array}$ & $\begin{array}{l}\text { Impact on lipase } \\
\text { expression and } \\
\text { activity }\end{array}$ & $\begin{array}{l}\text { Impact on HDL } \\
\text { cholesterol levels }\end{array}$ & Outcome for atherosclerosis or CVD & Reference \\
\hline \multirow[t]{6}{*}{ HL } & $\begin{array}{l}\text { S267F, } \\
\text { C-480T }\end{array}$ & $\begin{array}{l}2879 \text { cases and } \\
7735 \text { controls }\end{array}$ & ND & Increased & No association with risk of ischemic CVD & {$[8]$} \\
\hline & $\mathrm{C}-514 \mathrm{~T}$ & 501 & ND & ND & Increased coronary plaque area in carriers & {$[21]$} \\
\hline & $\begin{array}{r}\text { G-216A, } \\
\text { C-480T, } \\
\text { A-719G }\end{array}$ & $\begin{array}{l}1741 \text { cases and } \\
7948 \text { controls }\end{array}$ & ND & Increased & $\begin{array}{l}\text { Increased risk of ischemic heart disease in } \\
\text { homozygotes for all } 3 \text { variants }\end{array}$ & {$[22]$} \\
\hline & $\mathrm{C}-514 \mathrm{~T}$ & $\begin{array}{r}562 \text { cases and } \\
642 \text { controls }\end{array}$ & ND & Increased & $\begin{array}{l}\text { Increased risk of cardiovascular heart disease in } \\
\text { male carriers but not in female carriers }\end{array}$ & {$[23]$} \\
\hline & C-514T & $\begin{array}{l}496 \text { cases and } \\
294 \text { controls }\end{array}$ & $\begin{array}{l}\text { Decreased HL } \\
\text { activity }\end{array}$ & $\begin{array}{l}\text { Increased or not } \\
\text { affected }\end{array}$ & No association with risk of CVD & {$[24]$} \\
\hline & C-480T & 87 & ND & ND & $\begin{array}{l}\text { Increased carotid intima-media wall thickness } \\
\text { in homozygous carriers }\end{array}$ & {$[25]$} \\
\hline \multirow[t]{4}{*}{ EL } & Thr111Ile ${ }^{\mathrm{a}}$ & $\begin{array}{r}107 \text { cases and } \\
107 \text { controls }\end{array}$ & ND & Not affected & $\begin{array}{l}\text { Decreased risk of acute myocardial infarction } \\
\text { in carriers }\end{array}$ & {$[47]$} \\
\hline & Thr111Ile & $\begin{array}{r}265 \text { cases and } \\
265 \text { controls }\end{array}$ & ND & Increased & $\begin{array}{l}\text { Decreased risk of coronary artery disease in } \\
\text { carriers }\end{array}$ & [48] \\
\hline & Thr111Ile & $\begin{array}{l}1501 \text { cases and } \\
2639 \text { controls }\end{array}$ & ND & Not affected & $\begin{array}{l}\text { No association with risk of coronary heart } \\
\text { disease }\end{array}$ & [49] \\
\hline & Thr111Ile & $\begin{array}{l}607 \text { cases and } \\
1271 \text { controls }\end{array}$ & ND & Increased & $\begin{array}{l}\text { No association with risk of coronary artery } \\
\text { disease }\end{array}$ & {$[50]$} \\
\hline
\end{tabular}

$C V D$ cardiovascular disease, $H L$ hepatic lipase, $E L$ endothelial lipase, $N D$ not determined

a Please note that functional studies indicated that the Thr111Ile variant of endothelial lipase does not differ in expression and activity from wild-type endothelial lipase [34••]

genotype of the C-480T variant, who have higher HL activity, had a $13 \%$ greater carotid intima wall-thickness [25]. Taken together, these data stress the need for further carefully conducted studies that, in addition to genotyping the participating subjects, also aim to correlate HL mass as well as activity with relevant hard clinical end points of atherosclerotic CVD.

\section{Endothelial Lipase}

EL, encoded by the $L I P G$ gene, is a $68-\mathrm{kD}$ glycoprotein that was cloned independently in 1999 by two groups (Table 1) $[26,27]$. The amino acid sequence of EL is $41 \%$ homologous to HL. Nevertheless, EL is distinct from other members of the triacylglycerol family because it is synthesized and secreted by endothelial cells. In addition, expression of EL has been detected in human placenta, thyroid gland, lung, liver, kidney, ovary, testis, and macrophages in vitro as well as in foam cells within human atherosclerotic plaques (Table 1) [3, 26, 27]. EL has primarily sn-1phospholipase activity toward all lipoprotein subclasses, however, the preferred substrate is apparently phospholipids within HDL (Table 1) [3]. Similar to HL, EL mediates bridging between lipoproteins and cell surface heparin sulfate proteoglycans [3].

\section{EL and HDL Metabolism}

EL is a negative regulator of plasma HDL cholesterol levels (Table 1). Adenovirus-mediated overexpression of EL resulted in a marked decrease in plasma HDL cholesterol and apoA-I levels due to a dose-dependent increase in the catabolic rate of HDL apolipoproteins as well as HDL-CE [27, 28•, 29]. These metabolic effects are almost entirely dependent on the catalytic activity of EL and are not so much mediated by its bridging function, as overexpression of catalytically inactive EL had discernable effects on plasma HDL cholesterol only in HL-deficient mice but not in wild-type or apoA-I transgenic mice [3]. Conversely, loss-of-function studies in mice using either specific EL knockout models or antibody-mediated EL inhibition showed increased plasma levels of phospholipids, apoA-I, and HDL cholesterol due to a slower catabolic rate $[3,6 \bullet]$. Also a shift in the HDL size toward larger particles was noted [3]. Interestingly, inhibition of EL activity [3] or EL knockout [6•] in HL-deficient mice resulted in a further significant increase in plasma HDL cholesterol levels, indicating an independent and additive role of these lipases in HDL metabolism.

Based on these experimental findings, we have proposed a model in which hydrolysis of HDL phospholipids by EL destabilizes the HDL particle, resulting in shedding of 
poorly lipidated apoA-I molecules that are rapidly cleared by the kidneys (Fig. 1) [3, 29]. Concomitantly, the CE within the remainder of the HDL particle is rendered more susceptible toward SR-BI-mediated selective uptake, a mechanism that is also a prerequisite for EL-induced HDL remodeling to continue $[28 \cdot, 29]$. This mechanism results in an increased hepatic cholesterol content without translating into altered biliary cholesterol secretion [30].

To put these data from experimental mouse models into perspective, an important question is if EL has a similar impact on HDL metabolism in humans and thereby might represent a potential therapeutic target. In a sample of 510 healthy individuals with a family history of premature coronary artery disease, post-heparin plasma mass levels of EL were positively correlated with small HDL and negatively with large HDL particles [31]. Selected common polymorphisms of EL were also found to be associated with HDL phenotypes [32, 33]. However, the strongest data supporting a negative impact of EL on plasma HDL cholesterol in humans come from a recent report demonstrating that the low-frequency Asn396Ser LIPG variant results in reduced lipolytic activity and is strongly associated with increased plasma HDL cholesterol levels [34••]. Another rare mutation in the EL gene, G26S, impairs secretion of the EL protein, leading to reduced EL plasma levels and thereby also to elevated plasma HDL cholesterol in African-American carriers [35]. However, the more common Thr111Ile EL variant, which had previously been variably and inconsistently associated with plasma HDL cholesterol phenotypes [36, 37], did not impact on EL function in vitro and failed to correlate with plasma HDL cholesterol levels in a comprehensive meta-analysis of five cohort studies $[34 \bullet \cdot$.

\section{Impact of EL on Selected Key Steps of RCT}

Only a few studies have examined the effect of EL on HDL-mediated cellular cholesterol efflux (the first step in RCT), and none have achieved conclusive results (Table 1). Decreased EL expression in vitro in macrophages caused a decline in efflux toward apoA-I (i.e., the part of the pathway mainly mediated by ABCA1), whereas overexpression of EL or addition of exogenous EL had an opposite effect [38]. Notably, both abolishing the catalytic activity or the bridging function of EL partially inhibited macrophage cholesterol efflux [38], indicating that these two properties of EL are relevant in this process. In contrast, using modification of the efflux acceptors by EL, one study noted no effect on the ABCA1-dependent pathway [39], whereas another group reported a $63 \%$ increase in ABCA1-mediated efflux toward HDL from EL-overexpressing mice [40]. However, increased efflux toward plasma and HDL from EL knockout mice has also been observed, and an additive effect was seen in EL/HL double-knockout mice [6•]. With respect to SR-BI-mediated efflux, a reduced capacity of EL-modified HDL [39] or of serum from EL-overexpressing mice [40] was described.

Although it variably modulates cholesterol efflux, EL was consistently reported to enhance the second step in the RCT pathway, which is the uptake of HDL cholesterol into the liver (Table 1). While diminished selective uptake of CE was observed using $\mathrm{HDL}_{3}$ from EL-knockout mice [6•], SR-BI-mediated selective uptake was enhanced from HDL from EL-overexpressing mice in vitro and in vivo $[28 \cdot, 30]$. This increase in selective uptake is dependent upon both the catalytic activity and the bridging function of EL [41]. Consequently, these properties of EL resulted in vivo in a significant increase in hepatic cholesterol content following EL overexpression [28•, 30].

However, the major pathway of cholesterol excretion from the body, namely biliary sterol secretion either as free cholesterol or after metabolic conversion into bile acids, remained essentially unchanged in EL-overexpressing mice (Table 1). Correspondingly, EL overexpression did not affect the mass fecal output of neutral sterols and bile acids (Table 1) [30].

\section{EL and Macrophage-Specific RCT}

To date, two studies have explored the effect of EL on macrophage-specific RCT using ${ }^{3} \mathrm{H}$-cholesterol-labeled macrophage foam cells (Table 1). EL overexpression significantly decreased in vivo RCT and the same effect was observed with overexpression of profurin [42]. Profurin inhibits proprotein convertases, which in general mediate site-specific proteolysis and have specifically been shown to impact plasma HDL cholesterol levels via specific cleavage of EL resulting in inactivation of this lipase [42]; therefore, profurin overexpression has a similar metabolic effect than EL overexpression. In addition, angiopoietin-like 3 (ANGPTL3) has been demonstrated to be an endogenous inhibitor of EL, and consequently ANGPTL3 knockout mice have low plasma HDL cholesterol levels [3]. Therefore, the effect on RCT in ANGPTL3 knockouts would be expected to be the same as in mice overexpressing EL (namely, decreased), but this has not been experimentally tested thus far. On the other hand, overall RCT is unchanged in EL knockout mice [6 ${ }^{\bullet}$ ]. However, EL knockouts had increased plasma levels of cholesterol originating from macrophages during the time course of the study, and this increase was significantly greater than in HL knockout mice [6•]. No additive effect of the absence of both EL and HL on plasma counts or on overall RCT was observed $[6 \bullet]$, indicating that the effects 
of absence of EL and HL on overall RCT in mice are minor.

\section{EL and Atherosclerosis}

Studies exploring the role of EL in experimental atherogenesis have not yielded conclusive results thus far (Table 1). One study reported that targeted disruption of EL in apoE knockout mice attenuated atherosclerotic lesion formation by approximately $70 \%$ on chow and to a lesser degree on a Western-type diet [43]. Interestingly, the lack of EL resulted in increased plasma levels of antiatherogenic HDL as well as proatherogenic very low-density lipoprotein and LDL cholesterol [43]. Therefore, the authors rather proposed reduced monocyte adhesion to the vascular wall via heparan sulfate proteoglycans as an underlying mechanism for decreased atherosclerosis development in apoE/ EL double knockout mice [43]. On the contrary, in another comprehensive study, no differences in atherosclerotic lesion size and lesion macrophage content were detected between mice expressing or lacking EL either on the apoE knockout or the LDL-receptor knockout background [44]. Also, this report noted a slight but consistent increase in plasma levels of both cholesterol within HDL as well as apoB-containing lipoproteins [44].

Inflammation plays an important role in the development of atherosclerosis, and a link between EL and inflammation has been suggested. Expression of EL is substantially upregulated in cultured endothelial cells in response to proinflammatory cytokines and in humans during acute experimental inflammation [3]. Although EL has been shown to enhance monocyte adhesion to the vessel wall [43], another report suggested that EL decreases endothelial adhesion molecule expression by generating peroxisome proliferator-activated receptor $\alpha(\operatorname{PPAR} \alpha)$ ligands from HDL phospholipids [3]. Therefore, although EL expression is upregulated by inflammatory stimuli, it is currently unclear if EL itself exerts anti- or proinflammatory activity. In analogy to other secreted phospholipases [45], however, it could also be conceivable that EL expression induces endothelial dysfunction and thereby the susceptibility to atherosclerotic CVD, although this concept has not been experimentally addressed thus far.

In humans, the association between atherosclerotic cardiovascular disease and EL levels or variants of the EL gene has been investigated (summarized in Table 2). As indirect evidence, a significant positive correlation was found between coronary artery calcification scores and EL mass levels in pre-heparin (odds ratio $=1.67$ ) and post-heparin (odds ratio $=2.42$ ) plasma among apparently healthy individuals that persisted even after adjustment for plasma lipids, vasoactive medication, and established risk factors
[31]. A report on a relatively small group of patients with end-stage renal disease on hemodialysis suggested that during a 2-year follow-up, patients experiencing CVD events had higher serum EL levels compared with eventfree patients [46]. Two small case-control studies from Japan and China noted that the common Thr111Ile EL variant was related to a decreased risk of acute myocardial infarction and coronary artery disease, respectively $[47,48]$. However, the Thr111Ile variant was shown in in vitro assays to be indiscernible from wild-type EL [34••]. Subsequently, three larger Caucasian study populations could not confirm an impact of the Thr111Ile variant on cardiovascular risk [49]. More recent data from two independent, prospective, nestedcontrol studies reported that out of five single nucleotide polymorphism in $L I P G$, only the Thr111Ile variant was modestly associated with higher HDL cholesterol, higher apoA-I levels, and a higher concentration of larger HDL particles, whereas none of the EL variants tested proved to be a risk factor for atherosclerotic cardiovascular disease [50]. Clearly, more studies are needed that relate plasma levels and/or activity of EL to hard endpoints of CVD.

\section{Conclusions}

HL and EL are both negative regulators of plasma HDL cholesterol levels. For both lipases, variable effects on cholesterol efflux, which is the first step in RCT, have been reported. HL and EL increase hepatic cholesterol content, but this does not impact biliary sterol secretion. Overall, EL overexpression decreases macrophage-to-feces RCT, whereas EL as well as HL knockout did not affect RCT. In terms of establishing these lipases as targets for therapeutic intervention, increases in RCT upon inhibition are not likely to be expected; however, a decrease in RCT might not occur either. More work seems required on the impact of $\mathrm{HL}$ and EL on atherosclerotic disease, including measurements of plasma mass and activity. In addition, exploring other biological properties of these lipases, such as their role in modulating inflammatory pathways, might prove useful in deciding whether HL and EL are suitable targets for pharmacologic inhibition as a therapeutic strategy against atherosclerotic CVD.

Disclosure The authors report no potential conflicts of interest relevant to this article.

Open Access This article is distributed under the terms of the Creative Commons Attribution Noncommercial License which permits any noncommercial use, distribution, and reproduction in any medium, provided the original author(s) and source are credited. 


\section{References}

Papers of particular interest, published recently, have been highlighted as:

- Of importance

•. Of major importance

1. Baigent C, Keech A, Kearney PM, et al. Efficacy and safety of cholesterol-lowering treatment: prospective meta-analysis of data from 90,056 participants in 14 randomised trials of statins. Lancet. 2005;366:1267-78.

2. Lewis GF, Rader DJ. New insights into the regulation of HDL metabolism and reverse cholesterol transport. Circ Res. 2005;96:1221-32.

3. Yasuda T, Ishida T, Rader DJ. Update on the role of endothelial lipase in high-density lipoprotein metabolism, reverse cholesterol transport, and atherosclerosis. Circ J. 2010;74:2263-70.

4. Santamarina-Fojo S, Gonzalez-Navarro H, Freeman L, et al. Hepatic lipase, lipoprotein metabolism, and atherogenesis. Arterioscler Thromb Vasc Biol. 2004;24:1750-4.

5. Jin W, Marchadier D, Rader DJ. Lipases and HDL metabolism. Trends Endocrinol Metab. 2002;13:174-8.

6. - Brown RJ, Lagor WR, Sankaranaravanan S, et al. Impact of combined deficiency of hepatic lipase and endothelial lipase on the metabolism of both high-density lipoproteins and apolipoprotein B-containing lipoproteins. Circ Res. 2010;107:357-64. This is the first article determining the role of hepatic lipase and endothelial lipase knockout and hepatic lipase/endothelial lipase double-knockout on macrophage-to-feces reverse cholesterol transport.

7. Ruel IL, Couture P, Cohn JS, et al. Evidence that hepatic lipase deficiency in humans is not associated with proatherogenic changes in HDL composition and metabolism. J Lipid Res. 2004;45:1528-37.

8. Johannsen TH, Kamstrup PR, Andersen RV, et al. Hepatic lipase, genetically elevated high-density lipoprotein, and risk of ischemic cardiovascular disease. J Clin Endocrinol Metab. 2009;94:1264-73.

9. Isaacs A, Sayed-Tabatabaei FA, Njajou OT, et al. The $-514 \mathrm{C}->\mathrm{T}$ hepatic lipase promoter region polymorphism and plasma lipids: a meta-analysis. J Clin Endocrinol Metab. 2004;89:3858-63.

10. Amigo L, Mardones P, Ferrada C, et al. Biliary lipid secretion, bile acid metabolism, and gallstone formation are not impaired in hepatic lipase-deficient mice. Hepatology. 2003;38:726-34.

11. Barcat D, Amadio A, Palos-Pinto A, et al. Combined hyperlipidemia/ hyperalphalipoproteinemia associated with premature spontaneous atherosclerosis in mice lacking hepatic lipase and low density lipoprotein receptor. Atherosclerosis. 2006;188:347-55.

12. Dichek HL, Qian K, Agrawal N. The bridging function of hepatic lipase clears plasma cholesterol in LDL receptor-deficient "apoB48-only" and "apoB-100-only" mice. J Lipid Res. 2004;45:55160 .

13. Freeman L, Amar MJ, Shamburek R, et al. Lipolytic and ligandbinding functions of hepatic lipase protect against atherosclerosis in LDL receptor-deficient mice. J Lipid Res. 2007;48:104-13.

14. Qian K, Agrawal N, Dichek HL. Reduced atherosclerosis in chow-fed mice expressing high levels of a catalytically inactive human hepatic lipase. Atherosclerosis. 2007;195:66-74.

15. Mezdour H, Jones R, Dengremont C, et al. Hepatic lipase deficiency increases plasma cholesterol but reduces susceptibility to atherosclerosis in apolipoprotein E-deficient mice. J Biol Chem. 1997;272:13570-5.

16. Karackattu SL, Trigatti B, Krieger M. Hepatic lipase deficiency delays atherosclerosis, myocardial infarction, and cardiac dys- function and extends lifespan in SR-BI/apolipoprotein E double knockout mice. Arterioscler Thromb Vasc Biol. 2006;26:548-54.

17. Nong Z, Gonzalez-Navarro H, Amar M, et al. Hepatic lipase expression in macrophages contributes to atherosclerosis in apoEdeficient and LCAT-transgenic mice. J Clin Invest. 2003;112:367-78.

18. Hime NJ, Black AS, Bulgrien JJ, Curtiss LK. Leukocyte-derived hepatic lipase increases HDL and decreases en face aortic atherosclerosis in LDLr-/- mice expressing CETP. J Lipid Res. 2008;49:2113-23.

19. Hegele RA, Little JA, Vezina C, et al. Hepatic lipase deficiency. Clinical, biochemical, and molecular genetic characteristics. Arterioscler Thromb. 1993;13:720-8.

20. Dugi KA, Brandauer K, Schmidt N, et al. Low hepatic lipase activity is a novel risk factor for coronary artery disease. Circulation. 2001;104:3057-62.

21. Fan YM, Lehtimaki T, Rontu R, et al. The hepatic lipase gene C480T polymorphism in the development of early coronary atherosclerosis: the Helsinki Sudden Death Study. Eur J Clin Invest. 2007;37:472-7.

22. Andersen RV, Wittrup HH, Tybjaerg-Hansen A, et al. Hepatic lipase mutations, elevated high-density lipoprotein cholesterol, and increased risk of ischemic heart disease: the Copenhagen City Heart Study. J Am Coll Cardiol. 2003;41:1972-82.

23. Ji J, Herbison CE, Mamotte CD, et al. Hepatic lipase gene $-514 \mathrm{C} /$ $\mathrm{T}$ polymorphism and premature coronary heart disease. $\mathrm{J}$ Cardiovasc Risk. 2002;9:105-13.

24. Shohet RV, Vega GL, Anwar A, et al. Hepatic lipase (LIPC) promoter polymorphism in men with coronary artery disease. Allele frequency and effects on hepatic lipase activity and plasma HDL-C concentrations. Arterioscler Thromb Vasc Biol. 1999;19:1975-8.

25. Rundek T, Elkind MS, Pittman J, et al. Carotid intima-media thickness is associated with allelic variants of stromelysin-1, interleukin-6, and hepatic lipase genes: the Northern Manhattan Prospective Cohort Study. Stroke. 2002;33:1420-3.

26. Hirata K, Dichek HL, Cioffi JA, et al. Cloning of a unique lipase from endothelial cells extends the lipase gene family. J Biol Chem. 1999;274:14170-5.

27. Jaye M, Lynch KJ, Krawiec J, et al. A novel endothelial-derived lipase that modulates HDL metabolism. Nat Genet. 1999;21: 4248.

28. - Nijstad N, Wiersma H, Gautier T, et al. Scavenger receptor BImediated selective uptake is required for the remodeling of high density lipoprotein by endothelial lipase. J Biol Chem. 2009;284:6093-100. This is a comprehensive study including $H D L$ kinetics to demonstrate the complex interaction between EL, $H D L$, and the $S R-B I$.

29. Maugeais C, Tietge UJF, Broedl UC, et al. Dose-dependent acceleration of high-density lipoprotein catabolism by endothelial lipase. Circulation. 2003;108:2121-6.

30. Wiersma H, Gatti A, Nijstad N, et al. Hepatic SR-BI, not endothelial lipase, expression determines biliary cholesterol secretion in mice. J Lipid Res. 2009;50:1571-80.

31. Badellino KO, Wolfe ML, Reilly MP, Rader DJ. Endothelial lipase concentrations are increased in metabolic syndrome and associated with coronary atherosclerosis. PLoS Med. 2006;3:e22.

32. Yamakawa-Kobayashi K, Yanagi H, Endo K, et al. Relationship between serum HDL-C levels and common genetic variants of the endothelial lipase gene in Japanese school-aged children. Hum Genet. 2003;113:311-5.

33. Mank-Seymour AR, Durham KL, Thompson JF, et al. Association between single-nucleotide polymorphisms in the endothelial lipase (LIPG) gene and high-density lipoprotein cholesterol levels. Biochim Biophys Acta. 2004;1636:40-6.

34. •• Edmondson AC, Brown RJ, Kathiresan S, et al. Loss-of-function variants in endothelial lipase are a cause of elevated HDL cholesterol 
in humans. J Clin Invest. 2009;119:1042-50. This is an important study demonstrating that endothelial lipase is a negative regulator of HDL plasma levels in humans using an experimental approach that combines human genetics with in vitro work and proof-ofconcept studies in experimental animals.

35. Brown RJ, Edmondson AC, Griffon N, et al. A naturally occurring variant of endothelial lipase associated with elevated HDL exhibits impaired synthesis. J Lipid Res. 2009;50:19106.

36. Paradis ME, Couture P, Bosse Y, et al. The T111I mutation in the EL gene modulates the impact of dietary fat on the HDL profile in women. J Lipid Res. 2003;44:1902-8.

37. Halverstadt A, Phares DA, Ferrell RE, et al. High-density lipoprotein-cholesterol, its subfractions, and responses to exercise training are dependent on endothelial lipase genotype. Metabolism. 2003;52:1505-11.

38. Qiu G, Hill JS. Endothelial lipase promotes apolipoprotein AImediated cholesterol efflux in THP-1 macrophages. Arterioscler Thromb Vasc Biol. 2009;29:84-91.

39. Gauster M, Oskolkova OV, Innerlohinger J, et al. Endothelial lipase-modified high-density lipoprotein exhibits diminished ability to mediate SR-BI (scavenger receptor B type I)-dependent free-cholesterol efflux. Biochem J. 2004;382:75-82.

40. Yancey PG, Kawashiri MA, Moore R, et al. In vivo modulation of HDL phospholipid has opposing effects on SR-BI- and ABCA1mediated cholesterol efflux. J Lipid Res. 2004;45:337-46.

41. Strauss JG, Zimmermann R, Hrzenjak A, et al. Endothelial cellderived lipase mediates uptake and binding of high-density lipoprotein (HDL) particles and the selective uptake of HDL- associated cholesterol esters independent of its enzymic activity. Biochem J. 2002;368:69-79.

42. Jin W, Wang X, Millar JS, et al. Hepatic proprotein convertases modulate HDL metabolism. Cell Metab. 2007;6:129-36.

43. Ishida T, Choi SY, Kundu RK, et al. Endothelial lipase modulates susceptibility to atherosclerosis in apolipoprotein-E-deficient mice. J Biol Chem. 2004;279:45085-92.

44. Ko KW, Paul A, Ma K, et al. Endothelial lipase modulates HDL but has no effect on atherosclerosis development in apoE-/- and LDLR-/- mice. J Lipid Res. 2005;46:2586-94.

45. van der Giet $M$, Tolle $M$, Pratico D, et al. Increased type IIA secretory phospholipase $\mathrm{A}(2)$ expression contributes to oxidative stress in end-stage renal disease. J Mol Med. 2010;88:75-83.

46. Fujii H, Fukuda A, Tanaka M, et al. Putative role of endothelial lipase in dialysis patients with hypoalbuminemia and inflammation. Am J Nephrol. 2008;28:974-81.

47. Shimizu M, Kanazawa K, Hirata K, et al. Endothelial lipase gene polymorphism is associated with acute myocardial infarction, independently of high-density lipoprotein-cholesterol levels. Circ J. 2007;71:842-6.

48. Tang NP, Wang LS, Yang L, et al. Protective effect of an endothelial lipase gene variant on coronary artery disease in a Chinese population. J Lipid Res. 2008;49:369-75.

49. Jensen MK, Rimm EB, Mukamal KJ, et al. The T111I variant in the endothelial lipase gene and risk of coronary heart disease in three independent populations. Eur Heart J. 2009;30:1584-9.

50. Vergeer M, Cohn DM, Boekholdt SM, et al. Lack of association between common genetic variation in endothelial lipase (LIPG) and the risk for CAD and DVT. Atherosclerosis. 2010;211:558-64. 